University of Wollongong

Research Online

Faculty of Commerce - Papers (Archive)

Faculty of Business and Law

$14-9-2005$

\title{
MEBRS: A multiagent architecture for an experience based reasoning system
}

Zhaohao Sun

University of Wollongong, zsun@uow.edu.au

G. Finnie

Bond University

Follow this and additional works at: https://ro.uow.edu.au/commpapers

Part of the Business Commons, and the Social and Behavioral Sciences Commons

\section{Recommended Citation}

Sun, Zhaohao and Finnie, G.: MEBRS: A multiagent architecture for an experience based reasoning system 2005.

https://ro.uow.edu.au/commpapers/35

Research Online is the open access institutional repository for the University of Wollongong. For further information contact the UOW Library: research-pubs@uow.edu.au 


\title{
MEBRS: A multiagent architecture for an experience based reasoning system
}

\author{
Abstract \\ This paper reviews eight different inference rules for experience-based reasoning (EBR), and proposes a \\ multiagent architecture for an EBR system, which constitutes an important basis for developing any \\ multiagent EBR systems (EBRS). The proposed architecture consists of a global experience base (GEB), \\ and a multi-inference engine (MIE), which is the mechanism for implementing eight reasoning paradigms \\ based on eight inference rules for EBR. The proposed approach will facilitate research and development \\ of experience management, knowledge-based systems, and recognition of fraud and deception in e- \\ commerce.

\section{Keywords} \\ Experience-based reasoning, experience management, knowledge-based system, multiagent system \\ Disciplines \\ Business | Social and Behavioral Sciences

\section{Publication Details} \\ Sun, Z. \& Finnie, G. (2005). MEBRS: A multiagent architecture for an experience based reasoning system. \\ In Khosla R., Howlett, R.J., \& Jain L.C. (eds), Knowledge-Based Intelligent Information and Engineering \\ Systems: Proceedings of the 9th International Conference (KES2005) (pp. 972-978). Berlin, Germany: \\ Springer Verlag.
}




\title{
MEBRS: A Multiagent Architecture for an Experience Based Reasoning System
}

\author{
Zhaohao Sun, Gavin Finnie* \\ School of Economics and Information Systems, \\ University of Wollongong, Wollongong NSW 2522 Australia \\ Email: zsun@uow.edu.au \\ * Faculty of Information Technology, Bond University, Gold Coast Qld 4229 Australia \\ Email: gfinnie@staff.bond.edu.au
}

Abstract: This paper reviews eight different inference rules for experience-based reasoning (EBR), and proposes a multiagent architecture for an EBR system, which constitutes an important basis for developing any multiagent EBR systems (EBRS). The proposed architecture consists of a global experience base (GEB), and a multi-inference engine (MIE), which is the mechanism for implementing eight reasoning paradigms based on eight inference rules for EBR. The proposed approach will facilitate research and development of experience management, knowledge-based systems, and recognition of fraud and deception in e-commerce.

Keywords: Experience-based reasoning, experience management, knowledge-based system, multiagent system.

\section{Introduction}

Experience-based reasoning (EBR) is a reasoning paradigm based on logical arguments [2]. EBR as a technology has been used in many applications [13]. For example, EBR has been used in help desk systems to adapt to new business situations by "learning" from experience, tailoring a help desk to effectively maintain critical business systems [17][18]. However, the existing applications and studies have not been based on any firm theoretical foundations, because there are few studies on EBR from a logical viewpoint [9][12], although there are a lot of empirical works on EBR mainly in the business fields. How to automate experience remains a big issue. Taking into account research and development of case-based reasoning (CBR), Sun and Finnie [13][12] proposed eight different inference rules for EBR from a logical viewpoint, which cover all possibilities of EBR. However, how to implement an EBR system, and what is an experience-based inference engine based on the proposed eight inference rules for EBR [11][13] are still open problems. This paper attempts to fill the above gap by providing a multiagent architecture for an EBR system (for short, MEBRS), which is a core part for any multiagent EBR system. More specifically, this paper reviews eight different inference rules for EBR. Then the paper examines the multiagent architecture, MIE, and some other agents in the MEBRS. The rest of this paper is organized as follows: Section 2 reviews inference rules for EBR from a logical viewpoint. Section 3 proposes an architecture for EBR systems. Section 4 examines the multiagent framework for experience based inference engine. Section 5 looks at some intelligent agents in the MEBRS. Section 6 ends this paper with some concluding remarks. 


\section{Inference Rules for Experience Based Reasoning}

From a logic viewpoint, there are eight basic inference rules for performing EBR [13], which are summarized in Table 1, and cover all possible EBRs, and constitute the fundamentals for all EBR paradigms [9][11][13]. The eight inference rules are listed in the first row, and their corresponding general forms are shown in the second row respectively. Because four of them, modus ponens (MP), modus tollens (MT), abduction and modus ponens with trick (MPT) [10] are well-known in AI and computer sciences [8][7][11], we do not go into them any more, and focus on reviewing the other four inference rules in some detail. First of all, we illustrate modus tollens with trick (MTT) with an example. We have the knowledge in the knowledge base (KB):

1. If Klaus is human, then Klaus is mortal

2. Klaus is immortal.

What we wish is to prove "Klaus is human". In order to do so, let

- $P \rightarrow Q$ : If Klaus is human, then Klaus is mortal

- $P$ : Klaus is human

- $Q$ : Klaus is mortal.

Therefore, we have $P$ : Klaus is human, based on MTT, and the knowledge in the $\mathrm{KB}$ (note that $\neg Q$ : Klaus is not mortal). From this example, we can see that MTT is a kind of EBR.

Abduction with trick (AT) can be considered as a "dual" form of abduction, which is also the summary of a kind of EBR [13]. Abduction can be used to explain that the symptoms of the patients result from specific diseases, while abduction with trick can be used to exclude some possibilities of the diseases of the patient [12]. Therefore, abduction with trick is an important complementary part for performing system diagnosis and medical diagnosis based on abduction.

Inverse modus ponens (IMP) is also a rule of inference in EBR [13]. The "inverse" in the definition is motivated by the fact that the "inverse" is defined in mathematical logic: "if $\neg p$ then $\neg q$ ", provided that if $p$ then $q$ is given [3]. Based on this definition, the inverse of $P \rightarrow Q$ is $\neg P \rightarrow \neg Q$, and then from $\neg P, \neg P \rightarrow \neg Q$ we have $\neg Q$ using modus ponens. Because $P \rightarrow Q$ and $\neg P \rightarrow \neg Q$ are not logically equivalent, the argument based on IMP is not valid in mathematical logic. However, the EBR based on IMP is a kind of common sense reasoning, because there are many cases that follow IMP. For example, if John has enough money, then John will fly to China. Now John does not have sufficient money, then we can conclude that John will not fly to China.

The last inference rule for EBR is inverse modus ponens with trick (IMPT) [13]. The difference between IMPT and IMP is again "with trick", this is because the reasoning performer tries to use the trick of "make a feint to the east and attack in the west"; that is, he gets $Q$ rather than $\neg Q$ in the inverse modus ponens.

So far, we have reviewed eight different inference rules for EBR (see Table 1) from a logical viewpoint, four of them have been thoroughly used in computer science, mathematics, mathematical logic, philosophy and other sciences. The rest have not been appeared in any publications except [11][13], to our knowledge. However, they 
are all the abstraction and summary of experience or EBR in real world problems. Therefore, any research and development of each listed inference model is significant for understanding of intelligence, logic, EBR.

Table 1: Experience-based reasoning: Eight inference rules.

\begin{tabular}{|c|c|c|c|c|c|c|c|}
\hline MP & MT & $\begin{array}{l}\text { abdu- } \\
\text { ction }\end{array}$ & MTT & AT & MPT & IMP & IMPT \\
\hline$P$ & $\neg Q$ & $Q$ & $\neg Q$ & $Q$ & $P$ & $\neg P$ & $\neg P$ \\
\hline$\frac{P \rightarrow Q}{\therefore Q}$ & $\frac{P \rightarrow Q}{\therefore \neg P}$ & $\frac{P \rightarrow Q}{\therefore P}$ & $\frac{P \rightarrow Q}{\therefore P}$ & $\frac{P \rightarrow Q}{\therefore \neg P}$ & $\frac{P \rightarrow Q}{\therefore \neg Q}$ & $\frac{P \rightarrow Q}{\therefore \neg Q}$ & $\frac{P \rightarrow Q}{\therefore Q}$ \\
\hline
\end{tabular}

It should be noted that the inference rules "with trick" such as MTT, AT, MT and IMPT are non-traditional inference rules. However, they are really abstractions of some experience-based reasoning, although few have tried to formalize them. The "with trick" is only an explanation for such models. One can give other explanations for them. For example, one can use fraud or deception to explain them [13]. One can also use inference rules "with exception" to explain them.

\section{An Architecture of EBR Systems}

The architecture of an EBRS consists of experience users, $U$, which are either human customers or intelligent agents delegated by the human users or other systems, as shown in Fig. 1. The EBRS mainly consists of a user interface, a global experience

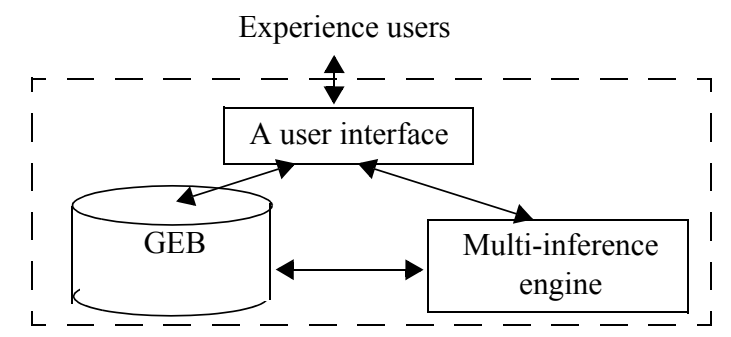

Fig. 1. A general architecture of an EBRS.

base (GEB) and a multi-inference engine (MIE). The user interface consists of some kind of natural language processing systems that allows the user to interact with the EBRS [7] (p. 282). The GEB, like the experience factory [1] and case base [11], consists of all the experiences that the system collects periodically, and the adapted new experiences retained when the system is running. The MIE is a multi-inference engine which consists of the mechanism for implementing eight reasoning paradigms based on eight inference rules for EBR with manipulating the GEB to infer experience requested by the experience user. The remarkable difference between the mentioned EBRS and the traditional KBS lies in that the latter's inference engine is based on a unique reasoning paradigm (or inference rule), while the MIE is based on many different reasoning paradigms. 


\section{MEBIE: A Multiagent Framework for Experience Based Inference Engine}

As mentioned in the previous section, the MIE is a multi-inference engine for EBR. MIE could automatically adapt itself to the changing situation and perform one of the mentioned inference rules for EBR. However, any existing intelligent system has not reached such a high level. The alternative strategy is to use multiagent technology to implement the MIE.

Multiagent systems (MAS) have been studied in the field of distributed AI (DAI) for more than 20 years [11][15]. Today these systems are not simply a research topic, but are also an important subject of academic teaching and industrial and commercial application. Recently, the term MAS is used for all types of systems composed of multiple agents showing the following characteristics:

- Each agent has incomplete capabilities to solve a problem

- There is no global system control over agents

- Data are decentralized

- Computation is asynchronous.

Rationality is a compelling notion in MAS. An ideal rational agent is defined as follows: for each possible percept sequence, it acts to maximize its expected utility, on the basis of its knowledge and the evidence from the percept sequence [5] (pp. 3-4).

Autonomy is also an important property of intelligent agents [5], in particular for mobile agents, which can move from one location to another while preserving their internal state [11]. Autonomy is the ability of agents to handle human user-defined tasks independently of the user and often without the user's guidance or presence [11].

Based on the above discussion, we propose a multiagent framework for an experience-based inference engine (for short MEBIE), which is a core part of a multiagent EBR system (MEBRS), as shown in Fig. 2. In this framework, eight rational agents (from MP agent to AbT agent) are semi-autonomous [11]. These eight agents are mainly responsible for performing EBR corresponding to eight inference rules in the EBRS respectively. In what follows, we discuss each of them in some detail.

1. The MP agent in the MEBIE is responsible for manipulating the GEB based on modus ponens to infer the experience requested by the experience user. This agent can be considered as an agentization of an inference engine in a traditional KBS in the EBRS. The function of the MP agent can be extended to infer the experience in the GEB based on fuzzy modus ponens [16] and similarity-based modus ponens [11]. It should be noted that the function of following mentioned agents can be also extended to infer the experience in the GEB based on corresponding fuzzy or similarity-based inference rules for EBR [13]

2. The MT agent manipulates the GEB to infer the experience requested by the experience user based on modus tollens, which is an inference rule discussed in AI

3. The Ab agent is responsible for manipulating the GEB to infer the experience requested by the experience user based on abductive reasoning [6]. This agent can generate the explanation for the experience inferred by the MEBIE 
4. The MPT agent is responsible for manipulating the GEB to infer the experience requested by the experience user based on modus ponens with trick. This agent can perform reasoning with trick [9], therefore the understanding of the experience provided by this agent requires special attention under some situations

5. The IMPT agent manipulates the GEB to infer the experience requested by the experience user based on inverse modus ponens with trick. This agent can also perform reasoning with trick [10], therefore the understanding of the experience provided by this agent requires special attention

Experience user

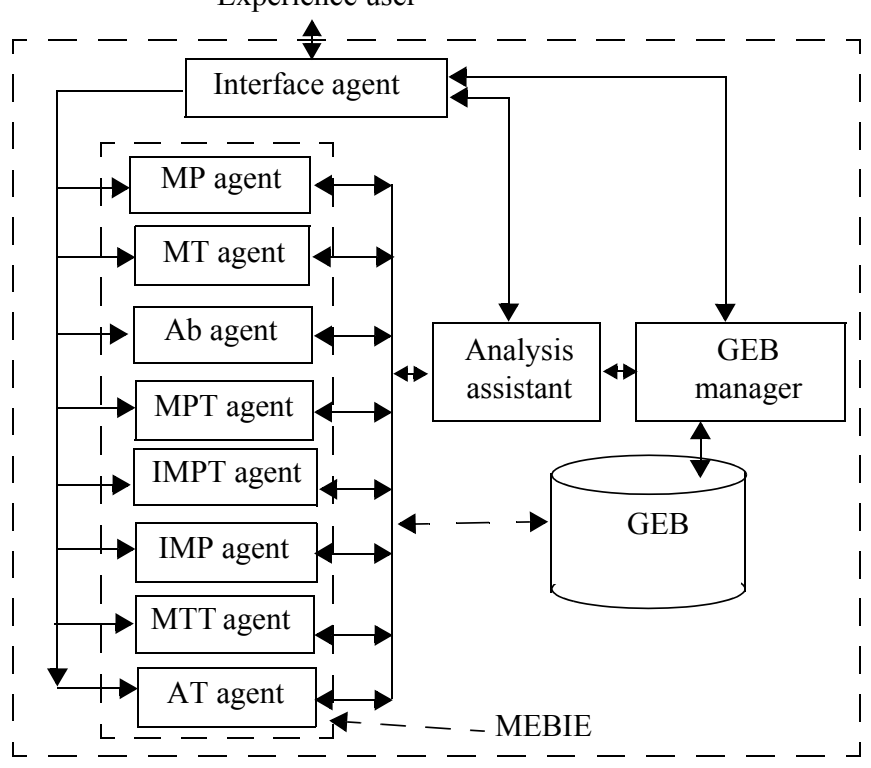

Fig. 2. MEBIE in a MEBRS.

6. The IMP agent is responsible for manipulating the GEB to infer the experience requested by the experience user based on inverse modus ponens (see Section 2)

7. The MTT agent manipulates the GEB to infer the experience requested by the experience user based on modus tollens with trick. This agent can also perform reasoning with trick [13], therefore the understanding of the experience provided by this agent also requires special attention

8. The $A T$ agent manipulates the GEB to infer the experience requested by the experience user based on abduction with trick. This agent can also perform reasoning with trick [13], therefore the understanding of the experience provided by this agent requires special attention.

\section{Some Other Agents in MEBRS}

For the proposed MEBRS there are some other intelligent agents, shown in Fig. 2. These are an interface agent, an analysis assistant and a GEB manager. In what follows, we will look at them in some depth. 
The Interface agent is an advisor to help the experience user to know which rational agent $\mathrm{s} /$ he likes to ask for help. Otherwise, the interface agent will forward the problem of the experience user to all agents in the MEBIE for further processing.

The output of the experience provided by the MEBIE can be considered as a suboutput, the final output as the solutions to the problem of the experience user will be processed with the help of the Analysis agent. Since different agents in the MEBIE use different inference rules, and then produce different, conflicting results with knowledge inconsistency. How to resolve such knowledge inconsistency is a critical issue for the MEBRS. This issue will be resolved by the Analysis assistant of the MEBRS. The Analysis assistant will

- Rank the degree of importance of the suboutputs from the MEBIE taking into account the knowledge inconsistency

- Give an explanation for each of the outputs from the MEBIE and how the different results are conflicting

- Possibly combine or vote to establish the best solutions

- Forward them to the interface agent who then forwards them to $U$.

The GEB manager is responsible for administering the GEB. Its main tasks are GEB creation and maintenance, experience evaluation, experience reuse, experience revision, and experience retention. Therefore the functions of the GEB manager are an extended form of the functions of a CBR system [11], because case base creation, case retrieval, reuse, revision and retention are the main tasks of the CBR system [4].

Now let us have a look at how the MEBRS works. The experience user, $U$, asks the interface agent to solve the problem, $p$. The interface agent asks $U$ whether a special reasoning agent should be need. $U$ does not know. Thus, the interface agent forwards $p$ (after formalizing it) to all agents in the MEBIE for further processing. The agent $i$ in the MEBIE manipulates the experience in the GEB based on $p$, and the corresponding reasoning mechanism, and then obtains the solution $s_{i}$, which is forwarded to the Analysis assistant. After the Analysis assistant receives all solutions $s_{i}$, $i=\{1, \ldots, 8\}$ to $p$, it will rank the degree of importance of the solutions, give an explanation for each of the solutions and how the results are conflicting, and then forward them (with $p$ ) to the interface agent who would then forward them to $U$. If $U$ accepts one of the solutions to the problem, then the MEBRS completes this experience transaction. In this case, the GEB manager will look at whether this case is a new experience. If yes, then it will add it to the GEB. Otherwise, it will keep some routine record to update the GEB. If $U$ does not accept the solution provided, the interface agent will ask $U$ to adjust some aspects of the problem $p$, which is changed into $p^{\prime}$, then the interface agent will once again forward the revised problem $p^{\prime}$ to the MEBIE for further processing.

\section{Concluding Remarks}

This paper first examined eight different inference rules for EBR. Then the paper proposed a multiagent architecture for an EBR system (MEBRS), and looked at the 
MEBIE and some other agents in the MEBRS. The proposed approach will facilitate research and development of EBR systems, knowledge based systems.

In future work, we will develop a prototype system for a multiagent system development environment for EBR system. To this end, we will further examine the internal mechanisms of the Analysis assistant such as how the agent ranks the degree of importance of the solutions produced by agents. We will also examine the communication, coordination, and cooperation among the agents with the proposed multiagent architecture.

\section{References}

[1] Bergmann R. Experience Management: Foundations, Development Methodology and Internet-Based Applications. LAIN 2432. Berlin: Springer, 2002

[2] Bosch. http://www.cs.tut.fi/ ohar/Slides2001/Bosch/tsld027.htm, 2001

[3] Epp SS. Discrete Mathematics with Applications, Brooks/Cole Publishing Company Pacific Grove, 1995, $801 \mathrm{p}$.

[4] Finnie G, Sun Z. $R^{5}$ model of case-based reasoning. Knowledge-Based Syst. 16(1) 2003, 5965

[5] Huhns MN, Singh MP (eds) Readings in Agents. San Francisco: Morgen Kaufmann Publishers, Inc. 1998

[6] Magnani L. Abduction, Reason, and Science, Processes of Discovery and Explanation. New York: Kluwer Academic/Plenum Publishers, 2001

[7] Nilsson NJ. Artificial Intelligence. A New Synthesis. San Francisco, California: Morgan Kaufmann Publishers, Inc. 1998, 513 p.

[8] Russell S, Norvig P. Artificial Intelligence: A modern approach. Upper Saddle River, New Jersey: Prentice Hall, 1995

[9] Sun Z, Finnie G. Brain-like architecture and experience-based reasoning, In: Proc. 7th Joint Conf on Information Sciences (JCIS), September 26-30, 2003 Cary, North Carolina, USA. $1735-1738$

[10] Sun Z, Weber K. Turing test and intelligence with trick. In: Proc 8th Ireland Conf on AI (AI97), Londonderry, Ireland, 1997, 217-224

[11] Sun Z, Finnie G. Intelligent Techniques in E-Commerce: A Case based Reasoning Perspective, Heidelberg: Springer, $306 \mathrm{p}$

[12] Sun Z, Finnie G. Experience Based Reasoning: A similarity-based perspective, IEEE Trans on Knowledge and Data Engineering, 2004, under review

[13] Sun Z, Finnie G. Experience based reasoning for recognising fraud and deception. In: Proc. Inter Conf on Hybrid Intelligent Systems (HIS 2004), December 6-8, Kitakyushu, Japan, IEEE Press, 2004, pp 80-85

[14] Torasso P, Console L, Portinale L, Theseider D. On the role of abduction. ACM Computing Surveys, 27 (3), 1995, 353-355

[15] Weiss, G (ed.). Multiagent Systems: A modern approach to distributed artificial intelligence, Cambridge, Massachusetts, London: The MIT Press, 1999

[16] Zimmermann HJ. Fuzzy Set Theory and its Application (3rd Edn). Boston/Dordrecht/London: Kluwer Academic Publishers, 1996

[17] http://wm2003.aifb.uni-karlsruhe.de/workshop/w06/GWEM2003_CfP_english.html

[18] http://www.iese.fraunhofer.de/experience_management/. 